\title{
A POSSIBLE FOURTH PLASMA THROMBOPLASTIN COMPONENT
}

\author{
By THEODORE H. SPAET, PAUL M. AGGELER, AND BEVERLY G. KINSELL
}

(From the Department of Medicine, Stanford University School of Medicine, San Francisco, and the Veterans Administration Hospital, Oakland, Calif.)

(Submitted for publication January 14, 1954; accepted April 17, 1954)

Recent studies have shown that the clinical and laboratory findings of hemophilia can be duplicated by at least two other blood coagulation disorders of related nature. In each case, laboratory studies have revealed a prolonged whole blood clotting time, reduced utilization of prothrombin in the course of clotting, and correction of the defect by the addition of small amounts of normal plasma. In none of these newly reported cases has a circulating anticoagulant been demonstrated, and all had normal amounts of fibrinogen, prothrombin, and both prothrombin conversion accelerators. On the basis of such findings these new conditions, like hemophilia, may be considered to involve a disturbance in intrinsic thromboplastin formation of the blood.

That the condition traditionally designated, "hemophilia" is not a disorder of uniform nature was first suggested in 1947 by the studies of Pavlovsky (1) wherein it was found that the blood of certain "hemophiliacs" improved clotting in others. In 1950, Koller, Krüsi, and Luchsinger (2) reported on a family of bleeders whose blood was likewise corrective against the coagulation defect of classical hemophiliacs, and for the first time recognized that a new disorder accounted for this phenomenon. The investigations of Aggeler, White and their co-workers (3-5) on a patient of this type established the existence of a new plasma thromboplastic factor. The new factor was found to contrast sharply with antihemophilic factor (AHF) by its presence in serum as well as plasma, its stability to storage at refrigerator temperature, its complete adsorption by barium sulfate, and its precipitation from plasma only at concentrations of ammonium sulfate greater than 40 per cent. Moreover, AHF is found mainly in Cohn's fraction I; the new factor is in fractions III and IV.

Reports of cases probably similar in nature soon followed. The name, "thromboplastin component
(PTC) deficiency" was chosen for the disease by Aggeler and his co-workers (3). Biggs and associates (6) designated it "Christmas Disease" and Soulier and Larrieu (7) suggested the use of hemophilia A and B to differentiate the two disorders.

Recently, Rosenthal, Dreskin, and Rosenthal (8) described still another hemophilia-like condition which appears to be due to the deficiency of a third plasma thromboplastic factor. This third factor has been named "plasma thromboplastin antecedent (PTA)." The coagulation disorder in these patients is corrected by both hemophilic and PTC-deficient plasma, as well as by normal plasma and serum. PTA was found to be absent from the 0 to 20 per cent ammonium sulfate precipitate of normal plasma, but was present in the 20 to 33 per cent fraction. As in the case of PTC, PTA was absent from fraction I of Cohn. Although normal plasma which had been adsorbed with barium sulfate corrected clotting in PTA-deficient patients, a partially purified PTC preparation made from a barium sulfate eluate of normal plasma was also partially corrective.

The studies to be described in the present report suggest the existence of an additional new member of the plasma thromboplastin complex and a corresponding deficiency thereof, resulting in another hemophilia-like disorder.

Report of case: The patient, C. C., was a thirty-one year old white American male who had had frequent and severe epistaxes from the ages of seven to eighteen. During this period four dental extractions were each followed by consistent oozing from the gums lasting one to two months. At the age of eighteen the patient was in an airplane crash, and as a result of injuries required an amputation of the right leg below the knee. Ten days after this procedure the wound began to bleed, and permanent hemostasis was not obtained for four months. Four revisions of the stump were attempted in the succeeding six year period, and each of these procedures was accompanied by post-operative bleeding lasting up- 
wards of one month. Following the first revision blood transfusion therapy was attempted but no beneficial results were noted. Other than a liability to easy bruising, neither hemarthroses nor any other hemorrhagic manifestations were reported.

The past history revealed nothing of significance, but the patient stated that there were both male and female bleeders in his family, as shown in Figure 1.

Physical examination was unrevealing save for the wellhealed amputation stump. Routine laboratory studies were all within normal limits.

\section{METHODS}

Blood was collected as follows: Two syringes were used. A small amount of blood was aspirated into the first syringe, and this was discarded. With the needle left in the vein the second syringe was then applied, and the necessary blood withdrawn. Uncoated glassware was used throughout the study except as otherwise indicated. For the preparation of plasma, one part of 0.1 molar potassium oxalate was used to nine parts of blood. All clotting procedures were carried out at $37^{\circ} \mathrm{C}$.

Coagulation times of whole blood were performed by the Lee-White three-tube method, but the serum prothrombin determinations were obtained from a fourth tube which had not been tipped or otherwise manipulated. Plasma prothrombin was estimated according to the techniques of Ware and Stragnell (9), and by the Quick method (10). Serum prothrombin was determined four hours after clotting, by the method of Ware and Stragnell (9). Fibrinogen and labile factor were measured by the method of Quick (10), proconvertin according to Owren and Aas (11), and anti-thrombin as described by Kay, Hutton, Weiss, and Ochsner (12). Excessive antithromboplastic activity was excluded as accomplished by Lewis and Ferguson (13) using Difco thromboplastin in serial dilution. The thrombin generation test was performed according to the method of Pitney and Dacie (14). In this test plasma is recalcified, and at measured intervals aliquots of the mixture are added to tubes containing fibrinogen solution. The clotting time of each fibrinogen sample thus treated indicates the rate of thrombin generation in the recalcified plasma.

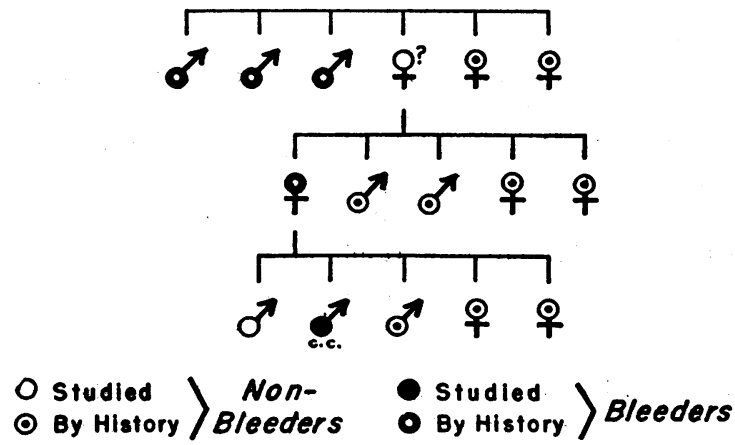

Fig. 1. Family Tree of Patient C. C.
TABLE I

Patient's blood coagulation profile

\begin{tabular}{lll}
\hline \hline & & \multicolumn{1}{c}{ Normal values } \\
\hline Platelets & $230,000 /$ cu. mm. & $200,000-400,000$ \\
Coagulation time & $22-32$ minutes & $8-20$ minutes \\
$\begin{array}{l}\text { Plasma Prothrombin } \\
\text { (Quick) }\end{array}$ & $90 \%$ & $80-110 \%$ \\
Plasma Prothrombin & & \\
(Ware and Stragnell) & $80-100 \%$ & $75-110 \%$ \\
Serum Prothrombin & $45-71 \%$ & $5-35 \%$ \\
Labile factor & $100 \%$ & $70-100 \%$ \\
Proconvertin & $100 \%$ & $70-120 \%$ \\
Fibrinogen & $625 \mathrm{mg} . \%$ & $250-400 \mathrm{mg} . \%$ \\
Clot retraction & normal & \\
Rumpel-Leede & negative & \\
\hline
\end{tabular}

Thromboplastin generation was studied by the technique of Biggs and Douglas (15). The basis of this test is the finding that in the presence of adequate calcium ion, a mixture of normal platelets, serum, and deprothrombinized plasma produces a potent thromboplastin. This thromboplastin can be measured by its activity in shortening the clotting time of normal recalcified plasma.

Platelet-poor native plasma was prepared from the patient's and normal blood in the following manner: All glassware was silicone-coated, and needles were treated with Arquadi. Processing was accomplished at $0^{\circ} \mathrm{C}$. The blood was first centrifuged at 2,500 rpm for ten minutes, and the plasma was pipetted into a second set of tubes. These were then spun at $30,000 \mathrm{rpm}$ in an International Refrigerated Centrifuge for thirty minutes. The resulting plasma, free of anticoagulants, was used promptly for tests of platelet function.

For the preparation of platelet suspensions, the blood was collected into silicone-coated glassware. Sequestrene was used as the anticoagulant, $0.4 \mathrm{cc}$. of a $10 \mathrm{per}$ cent solution having been used per $15 \mathrm{cc}$. of blood. The blood was first centrifuged at $1,000 \mathrm{rpm}$ for ten minutes, and the platelet-rich plasma separated. The platelets were collected by centrifugation at 3,000 rpm for thirty minutes, and washed twice in isotonic saline. They were finally suspended in a volume of saline such that a concentration of about 100,000 per cu. mm. resulted.

Platelet activity was determined by adding $0.9 \mathrm{cc}$. of native plasma to $0.1 \mathrm{cc}$. of platelet suspension. Four hours after clotting of this mixture, the prothrombin content of the resulting serum was measured as described above.

Both for measurement of activity. of various blood derivatives in correcting the patient's clotting defect, and for the determination of AHF, PTC, and PTA in the patient's blood, a similar technique was used. In each case, the appropriate plasma, serum, or protein fraction was diluted as indicated in Results, and $0.1 \mathrm{cc}$. was added to $1.9 \mathrm{cc}$. of the freshly drawn whole blood being used as the indicator of activity. The mixture was allowed to clot, and serum prothrombin measured after four hours of incubation. Studies on the clotting times of such mixtures were not undertaken because it was felt that the inaccuracy of the clotting time was too great to permit adequate interpretation. 


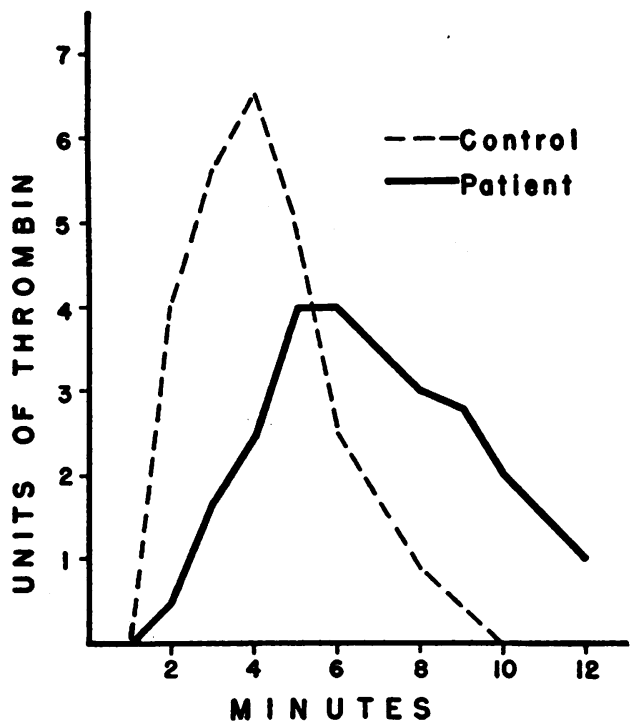

Fig. 2. Evolution of Thrombin in Patient's Recalcified Plasma Compared to Normal Control

The concentrated PTC preparation used in these studies was prepared by adsorption of Cohn's fraction IV with barium sulfate, and elution of this barium sulfate with sodium citrate (4).

The ability of the patient's plasma and serum to activate purified prothrombin, was kindly measured by Johnson, according to her recently published technique (16).

Ammonium sulfate fractionation of normal plasma was accomplished as outlined by White, Aggeler, and Glendening (4). The resulting plasma fractions and plasma fractions of Cohn ${ }^{1}$ were tested in concentrations proportional to their original plasma values.

\section{RESULTS}

A. Identification of a new coagulation disorder.

Coagulation of the patient's blood. Table I shows the results of clotting studies performed on the patient's blood. Of these tests, only the clotting time and the prothrombin consumption were abnormal. However, neither of these abnormalities was profound. A further reflection of the impaired blood coagulation was seen in the evolution of thrombin in recalcified plasma; the appearance of thrombin was delayed, and the peak concentration attained was somewhat below normal (Figure 2).

The clotting defect is more nicely demonstrated in the thromboplastin generation test, in which it is

\footnotetext{
${ }^{1}$ Kindly provided by the Cutter Laboratories, Berkeley, California.
}

evident that there is marked retardation of thromboplastin formation (Figure 3). Characteristic patterns were obtained by substituting singly, reagents prepared from the patient's blood. Abnormal curves were obtained when either the patient's deprothrombinized plasma or serum was used; but when his platelets alone were substituted, thromboplastin generation was normal. These findings differ from those of both hemophilia and PTC deficiency. In hemophilia thromboplastin generation is abnormal only when deprothrombinized plasma is substituted; in PTC deficiency the test is positive only when serum is substituted. In PTA deficiency any substitution produces improvement (17). The results of this test also indicate that platelet function was normal, and this finding is confirmed by other studies of platelet activity.

The activation of purified prothrombin by the patient's serum and plasma is shown in Figure 4. There is considerably reduced activity in the plasma, but the serum gives essentially a similar curve. This pattern is similar to that found in hemophilia, but differs from the one obtained in PTC deficiency where the serum is even less active than plasma.

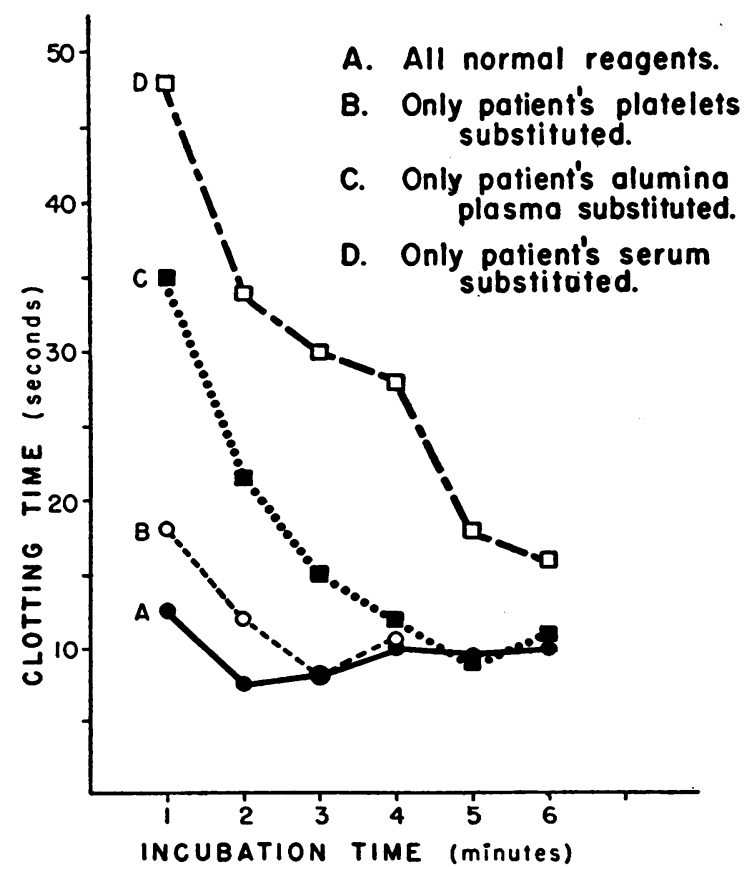

Fig. 3. Activity of Patient's Reagents when Substituted Singly in the Thromboplastin Generation TEST 
TABLE II

Studies of platelet function *

\begin{tabular}{lr}
\hline \multicolumn{1}{c}{ Mixture } & $\begin{array}{c}\text { Serum } \\
\text { prothrombin } \\
(\%)\end{array}$ \\
\hline Normal plasma + saline & 100 \\
Normal plasma + normal platelets & 24 \\
Normal plasma + patient's platelets & 12 \\
Patient's plasma + saline & $\mathbf{1 0 0}$ \\
Patient's plasma + normal platelets & $\mathbf{5 5}$ \\
\hline
\end{tabular}

* Residual serum prothrombin 4 hours after clotting, when $0.1 \mathrm{cc}$. of platelet suspension was added to $0.9 \mathrm{cc}$. of "native" plasma.

A test for antithromboplastin was negative (Table III). In each case the addition of the diluted thromboplastin was as effective against the patient's plasma as in the control. The antithrombin titer of both the patient's and control plasma was $1: 64$.

Platelet studies. As can be seen from Table I, platelets were normal in number, and clot retraction was adequate. That the platelets likewise had normal clotting activity is shown in Table II. The patient's platelets restored normal prothrombin consumption to normal, platelet-poor native plasma. In contrast, normal platelets added to the patient's platelet-poor native plasma did not result in comparable prothrombin consumption, although an excess of normal platelets was able to

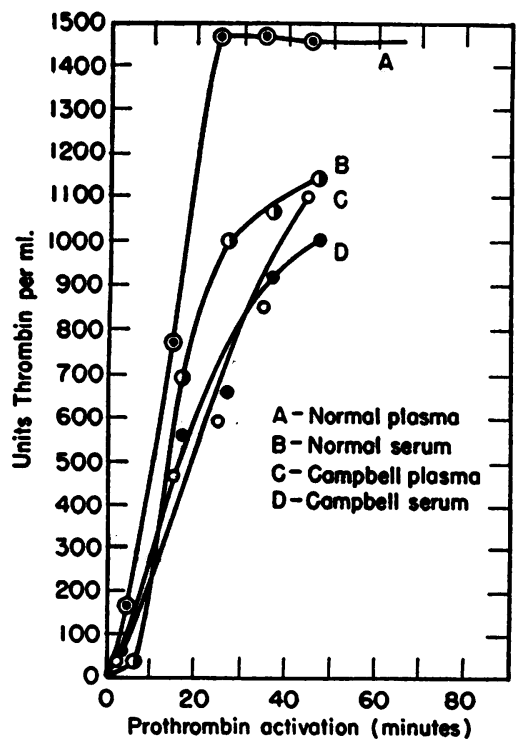

Fig. 4. Activation of Purified Prothrombin By 1:20 Dilutions of Patient's Plasma and Serum in the Presence of Platelets, Compared to Normal Control (Courtesy of Johnson, S. A. and Seegers, W. H.)
TABLE III

Clotting time in seconds, of patient's plasma when treated with diluted thromboplastin

\begin{tabular}{ccc}
\hline Reagent added & $\begin{array}{c}\text { Patient's } \\
\text { clotting } \\
\text { time }\end{array}$ & $\begin{array}{c}\text { Control } \\
\text { clotting } \\
\text { time }\end{array}$ \\
\hline $\begin{array}{c}\text { Thromboplastin } \\
\text { (undiluted) }\end{array}$ & 14.0 & 14.5 \\
$\begin{array}{c}\text { Thromboplastin } \\
(1: 100)\end{array}$ & 35.0 & 37.0 \\
$\begin{array}{c}\text { Thromboplastin } \\
(1: 200)\end{array}$ & 50.0 & 47.0 \\
\hline
\end{tabular}

improve clotting further. These findings are in conformity with those obtained in the thromboplastin generation test, and indicate that the clotting defect resides in the plasma rather than in the cellular elements of the blood.

Studies of previously described plasma thromboplastic factors. The activity of the patient's plasma in improving clotting of hemophilic, PTC-deficient, and PTA-deficient blood is shown in Table IV. In each case, the ability of the patient's plasma to improve prothrombin consumption compares favorably to that of normal plasma. Similarly, hemophilic, PTC-deficient, and PTA-deficient plasma and serum were effective in restoring normal prothrombin consumption to the patient's blood, as shown in Table V.

\section{B. Properties of the deficient factor.}

Stability. The stability of the clotting factor lacking in the present patient is shown in Table

TABLE IV

Activity of various plasmas and sera against patient's clotting disorder *

\begin{tabular}{lc}
\hline \multicolumn{1}{c}{ Preparation added } & $\begin{array}{c}\text { Improvement in } \\
\text { prothrombin } \\
\text { consumption } \\
\text { (\% control-\% test) }\end{array}$ \\
\hline Normal plasma & $22-38$ \\
Normal serum & 52 \\
Hemophilic plasma & 26 \\
PTC-deficient plasma & 28 \\
PTC-deficient serum & 35 \\
PTA-deficient plasma & 27 \\
Patient's plasma stored frozen & 0 \\
Patient's serum & 9 \\
Saline & 0
\end{tabular}

* One-tenth cc. of undiluted plasma or serum was added to $1.9 \mathrm{cc}$. of the patient's blood, and the serum prothrombin determined 4 hours after clotting. The control is the serum prothrombin of the patient's unmodified blood incubated at the same time. 
TABLE V

Activity of patient's plasma against thromboplastic disorders *

\begin{tabular}{lc}
\hline \multicolumn{1}{c}{ Combination of : } & $\begin{array}{c}\text { Serum } \\
\text { prothrombin } \\
(\%)\end{array}$ \\
\hline Hemophilic blood alone & 100 \\
2\% patient's plasma + hemophilic blood & 34 \\
2\% normal plasma + hemophilic blood & 42 \\
PTC-deficient blood alone & 80 \\
5\% patient's plasma + PTC-deficient blood & 24 \\
5\% normal plasma + PTA-deficient blood & 22 \\
Patient's plasma + PTA-deficient blood & Same as \\
& normalt
\end{tabular}

* In each case $0.1 \mathrm{cc}$. of the patient's plasma appropriately diluted was added to $1.9 \mathrm{cc}$. of test blood, and the serum prothrombin determined 4 hours after clotting.

† Rosenthal, R. L.: Personal communication.

VI. In each case, $0.1 \mathrm{cc}$. of undiluted plasma was added to $2 \mathrm{cc}$. of the patient's whole blood, and the serum prothrombin determined four hours after clotting. The activity of normal plasma is stable with storage for at least one week at room or refrigerator temperature; indeed, the activity of the plasma appears to be somewhat enhanced after storage. However, at $56^{\circ} \mathrm{C}$. it is rapidly destroyed. Thus, in its storage properties the new factor resembles PTC, although it is even less stable to heat. In contrast, AHF is extremely storage-labile, and relatively heat-stable. Comparable data are not available for the stability of PTA.

Fractionation behaviour. The activity of various preparations of nomal plasma is indicated in Table VII. The activity is not removed by repeated treatment of plasma with barium sulfate. Passage through a Seitz filter containing 50 per cent asbestos removes a considerable amount of activity, but those with lower concentrations have

TABLE VI

Stability of plasma activity *

\begin{tabular}{lc}
\hline \multicolumn{1}{c}{ Type of plasma } & $\begin{array}{c}\text { Improvement in } \\
\text { prothrombin } \\
\text { consumption } \\
\text { (\% control-\% test) }\end{array}$ \\
\hline Stored at $4^{\circ} \mathrm{C}$. for 1 week & 37 \\
Stored at $20^{\circ} \mathrm{C}$. for 1 week & 34 \\
Heated at $56^{\circ} \mathrm{C}$. for 5 minutes & 2 \\
\hline
\end{tabular}

* One-tenth cc. of plasma was added to $1.9 \mathrm{cc}$. of the patient's blood, and the serum prothrombin determined 4 hours after clotting. The control is the serum prothrombin of the patient's unmodified blood incubated at the same time.
TABLE VII

Actioity of plasma fractions *

\begin{tabular}{lc}
\hline Plasma fraction added & $\begin{array}{c}\text { Improve- } \\
\text { ment in } \\
\text { prothrombin } \\
\text { consumption } \\
\text { (\% control- } \\
\% \text { test) }\end{array}$ \\
\hline Barium sulfate plasma & 28 \\
Fraction I & 0 \\
Fraction II & 0 \\
Fraction III & 21 \\
Fraction IV & 30 \\
Fraction V & 0 \\
Seitz-filtered plasma (50\% asbestos) & 19 \\
PTC concentrate (8-fold concentrate) & 42 \\
PTC concentrate (reconstituted) & 10 \\
Precipitate from 25\% ammonium sulfate plasma & 8 \\
Precipitate from 33\% ammonium sulfate plasma & 23 \\
Precipitate from 50\% ammonium sulfate plasma & 30 \\
Supernatant from 50\% ammonium sulfate plasma & 10
\end{tabular}

* Each fraction was used in its normal plasma concentration. One-tenth cc. of the fraction was added to 0.9 cc. of the patient's blood and the serum prothrombin determined 4 hours after clotting. The control is the serum prothrombin of the patient's unmodified blood incubated at the same time.

little effect. In these respects its behaviour is similar to that of AHF.

Although the new factor differs from PTC with respect to its adsorption on barium sulfate, there is similarity in the Cohn fractions wherein they are found. Of the various fractions tested, only fractions III and IV were active. AHF, in contrast, is found in fraction I, and PTA in III and IV (17). Likewise, the new factor resembles PTC in that 50 per cent ammonium sulfate saturation is required for maximal precipitation from normal plasma. It differs from AHF and PTA which are found in the 33 per cent ammonium sulfate fraction (18). An unexpected finding was that of activity in a concentrated PTC preparation which was prepared from an eluate of barium sulfate used to adsorb fraction. IV.

Survival of the factor in vivo. It was anticipated that the corrective effect of normal plasma on the patient's blood clotting in vivo would be comparable to its in vitro activity. This has not proved to be the case. The following forms of transfusion therapy were tried : 250 and $500 \mathrm{cc}$. of fresh, citrated plasma, $100 \mathrm{cc}$. of heparinized serum, 350 cc. of whole blood by direct transfusion, and 100 cc. of 10 per cent fraction IV. In each case there was little or no change in either the patient's whole 
blood clotting time or prothrombin consumption. The minimal changes that were noted had all disappeared within an hour after completion of the transfusion.

\section{DISCUSSION}

The above data indicate that the following conclusions are justified: 1 ) The coagulation defect in our patient appears to result from defective plasma thromboplastin formation. This is reflected in the diminished utilization of prothrombin in the course of his blood clotting, and the retarded generation of thromboplastin in the Biggs and Douglas test. 2) The origin of the defect does not reside in the platelets; for the patient's platelets behave normally both in the thromboplastin generation test and in their ability to restore normal prothrombin consumption to "native" normal plasma. Moreover, in both of these situations, normal platelets failed to correct the patient's clotting defect. 3) The disorder does not result from lack of antihemophilic factor, as shown by several tests. Mixing the patient's blood with hemophiliac blood produces mutual correction of clotting in each case. In the thromboplastin generation test substitution of either the patient's serum or deprothrombinized plasma resulted in an abnormal curve, whereas in the hemophiliac it is only the plasma substitution which gives an abnormal result. The corrective activity of normal plasma for our patient was stable on storage, but labile to heat. AHF is storagelabile and relatively heat-stable. For our patient corrective activity was found in serum, Cohn's fractions III and IV only, and in the precipitate from 50 per cent ammonium sulfate saturated normal plasma. AHF is not found in serum, is mainly concentrated in Cohn's fraction I, and requires only 33 per cent ammonium sulfate for its precipitation from normal plasma. 4) The differentiation from PTC deficiency is likewise sharp. Here, too, there is mutual correction of the clotting defect with mixing of respective bloods. PTC deficiency also shows a characteristic and different pattern in the thromboplastin generation test in that it produces an abnormal curve only when serum is substituted. In the activation of purified prothrombin the present patient's plasma and serum were both equally defective; whereas in PTC deficiency the serum is even more defective than plasma. The activity of normal plasma against our patient's de- fect is not removed by adsorption with barium sulfate, or by low asbestos Seitz filtration; whereas PTC is completely removed by either of these procedures.

Differentiation of the present disorder from PTA deficiency is primarily based on the tests of mutual correction of one blood with plasma of the other. PTA activity is maximally precipitated with 33 per cent ammonium sulfate, and further differentiation is provided by the thromboplastin generation test in which substitution of either normal plasma or serum provides correction for PTA deficiency.

Clinically the thromboplastic disorders differ in their response to transfusion. In hemophilia the effect of blood or plasma is quite transient, usually lasting less than 24 hours. In PTC deficiency there is a considerably more prolonged effect, and coagulation tests may not return to control levels for three weeks following a transfusion (4). An intermediate response is seen in PTA deficiency (17) in that transfusion produces an effect lasting about one week. Our patient was quite refractory to any form of transfusion therapy.

On the basis of these findings, it would appear likely that there is an additional plasma thromboplastic component which is distinct from AHF, PTC, and PTA. However, the introduction of a new coagulation factor must be made with greatest caution, and artefact must be carefully excluded. In the present case, three such possibilities warrant serious consideration. 1) Exclusion of a deficiency in one of the previously known factors is based on the traditional method of measuring the ability of the patient's plasma or serum to correct clotting in known defective bloods. The underlying assumption in such a method is that there can be correction only by supplying the substance specifically lacking. Such an assumption does not admit the possibility of nonspecific correction, a simple example of which would be the corrective activity of trypsin on hemophilic blood (18). It has been our experience that prothrombin consumption in mixtures of normal bloods may exceed that obtained in either alone. As the clotting in our patient was only moderately abnormal, the activity of the other abnormal plasmas in correcting his defect could possibly have resulted from non-specific activity. On the other 
hand, it is less likely that his plasma could improve so markedly the severely abnormal bloods of the patients with known disorders, on a non-specific basis. At least in the cases of established hemophilia and PTC deficiency, such non-specific correction has not been found. 2) A combined but partial deficiency of two of the previously described factors could result in a situation by which addition of a blood defective in one of them would result in improved clotting. Such a case has actually been reported by Soulier and Larrieu (19), in which hemophilia was combined with PTC deficiency. However, each deficiency was clearly demonstrated by a suitable assay procedure. It would seem unlikely that a major defect of a factor would be missed by the present techniques, as the patient's plasma was used only in high dilution when tested against the other defective bloods. 3) The present syndrome could be produced by a circulating anticoagulant. The failure of transfusion to improve the patient's clotting underlines this possibility, although the active agent could, like prothrombin, be very rapidly metabolized (20). Such an hypothetical anticoagulant would have to be present in extremely low concentration, for as little as 1 per cent normal plasma produced appreciable improvement of the patient's prothrombin consumption in vitro. All attempts to demonstrate such an anticoagulant were unsuccessful, nor is it easy to see where in the coagulation process it could act without at least partially neutralizing one of the other known thromboplastic components. In some of the reported patients with circulating anticoagulants, it has been demonstrated that there is specific neutralization of AHF $(15,21)$. Nevertheless, these considerations indicate the need for independent confirmation before the suggested new coagulation factor can be accepted without reservation. This has been provided to a certain degree by the finding of an additional patient whose blood resembles that of ours. Rosenthal (17) has studied a blood evidently defective in thromboplastin formation which is corrected by plasma of patients with hemophilia, PTC deficiency and PTA deficiency, but not by the plasma of our patient.

On the assumption that a new coagulation factor has indeed been unearthed, the question of terminology is immediately presented. Several techniques of nomenclature are in current use. In one, the new disorder is given the name of the first family in which it was detected (Moena Anomaly, Christmas Disease). A second method attempts to apply descriptive terms to the missing factor as it appears to function in the clotting mechanism, thus: plasma thromboplastin component (PTC), plasma thromboplastin antecedent (PTA). Such terms imply that the place of the factors in the coagulation scheme is established, although the accompanying data are less conclusive. Moreover, this system does not have the flexibility to incorporate newly discovered factors. The system of nomenclature proposed by Soulier and Larrieu (7) is perhaps more satisfactory for the present needs. According to this scheme the various thromboplastic disorders are lettered, so that classical hemophilia is "hemophilia A," PTC deficiency is "hemophilia B," PTA deficiency is "hemophilia C," and the present disorder "hemophilia D." The difficulty involved in Soulier and Larrieu's system is the implication that all these conditions are variations of the same disease, whereas the data indicate that they are distinctly different disorders with similar clinical pictures. Therefore, Aggeler, White, and Spaet (22) prefer to retain the name "hemophilia" for the classical disease and append Greek numerical prefixes for the other thromboplastin deficiency diseases. PTC deficiency thus becomes deuterohemophilia, PTA deficiency tritohemophilia and the present disorder tetartohemophilia. These authors suggest a uniform nomenclature for the corresponding coagulation factors in which the generic term for the group is "plasma thromboplastic factor (PTF)." Each factor would be identified by an appended letter as follows: PTF-A (AHF), PTF-B (PTC), PTF-C (PTA), and PTF-D (present factor).

\section{SUMMARY}

A patient is reported with a blood clotting abnormality consisting of a prolonged coagulation time, reduced prothrombin utilization, and retarded generation of plasma thromboplastin. No anticoagulant was demonstrable, and all previously described coagulation factors were normally present. Normal plasma was corrective for the defect, and activity was found in serum, Cohn's fractions III and IV, and in the 50 per cent ammonium sulfate saturation precipitate of normal plasma. The 
activity was heat-labile, storage-stable, not adsorbed on barium sulfate, and removed only by high concentration asbestos Seitz filters. Transfusion of blood or its fractions did not improve the patient's clotting.

It is tentatively suggested that there is a previously undescribed coagulation factor concerned with the formation of plasma thromboplastin. It is proposed that this new factor be called, "plasma thromboplastic factor D (PTF-D)," and that its deficiency be named, "tetartohemophilia."

\section{REFERENCES}

1. Pavlovsky, A., Contribution to the pathogenesis of hemophilia. Blood, 1947, 2, 185.

2. Koller, F., Krüsi, G., and Luchsinger, P., Ueber eine besondere Form Hämorrhagischer Diathese. Schweiz. med. Wchnschr., 1950, 80, 1101.

3. Aggeler, P. M., White, S. G., Glendening, M. B., Page, E. W., Leake, T. B., and Bates, G., Plasma thromboplastin component (PTC) deficiency: A new disease resembling hemophilia. Proc. Soc. Exper. Biol. \& Med., 1952, 79, 692.

4. White, S. G., Aggeler, P. M., and Glendening, M. B., Plasma thromboplastin component (PTC). A hitherto unrecognized blood coagulation factor. Case report of PTC deficiency. Blood, 1953, 8, 101.

5. White, S. G., Aggeler, P. M., and Emery, B. E., Plasma thromboplastin component (PTC) potency of plasma fractions. Proc. Soc. Exper. Biol. \& Med., 1953, 83, 69.

6. Biggs, R., Douglas, A. S., MacFarlane, R. G., Dacie, J. V., Pitney, W. R., Merskey, C., and O'Brien, J. R., Christmas Disease. A condition previously mistaken for haemophilia. Brit. Med. J., 1952, 2, 1378.

7. Soulier, J.-P., and Larrieu, M.-J., Nouvelle méthode de diagnostic de l'hémophilie. Dosage des facteurs antihémophiliques A et B. Sang, 1953, 24, 205.

8. Rosenthal, R. L., Dreskin, O. H., and Rosenthal, N., New hemophilia-like disease caused by deficiency of a third plasma thromboplastin factor. Proc. Soc. Exper. Biol. \& Med., 1953, 82, 171.
9. Ware, A. G., and Stragnell, R., An improved one stage prothrombin method. Am. J. Clin. Path., 1952, 22, 791.

10. Quick, A. J., The Physiology and Pathology of Hemostasis, Philadelphia, Lea \& Febiger, 1951.

11. Owren, P. A. and Aas, K., The control of Dicumarol therapy and the quantitative determination of prothrombin and proconvertin. Scandinav. J. Clin. \& Lab. Invest., 1951, 3, 201.

12. Kay, J. H., Hutton, S. B., Jr., Weiss, G. N., and Ochsner, A., Studies on an antithrombin. III. A plasma antithrombin test for the prediction of intravascular clotting. Surg., 1950, 28, 24.

13. Lewis, J. H., and Ferguson, J. H., Hemorrhagic diathesis due to PTC (plasma thromboplastin component) deficiency. Proc. Soc. Exper. Biol. \& Med., 1953, 82, 445.

14. Pitney, W. R., and Dacie, J. V., A simple method of studying the generation of thrombin in recalcified plasma. Application in the investigation of haemophilia. J. Clin. Path., 1953, 6, 9.

15. Biggs, R., and Douglas, A. S., The thromboplastin generation test. J. Clin. Path., 1953, 6, 23.

16. Johnson, S. A., Activation of purified prothrombin with hemophilic plasma. Am. J. Clin. Path., 1953, 23, 875.

17. Rosenthal, R. L., Personal communication.

18. Tagnon, H. J., Effect of intravenous injection of trypsin on the blood coagulation time in hemophilia. Proc. Soc. Exper. Biol. \& Med., 1944, 57, 45.

19. Soulier, J. P., and Larrieu, M. J., Differentiation of hemophilia into two groups. A study of thirtythree cases. New England J. Med., 1953, 249, 547.

20. Blood Clotting and Allied Problems, Transactions of the Fifth Conference, 1952, ed. by J. E. Flynn, Josiah Macy Jr. Foundation, New York, 1952, p. 44.

21. Frick, P. G., Hemophilia-like disease following pregnancy. With transplacental transfer of an acquired circulating anticoagulant. Blood, 1953, 8, 598.

22. Aggeler, P. M., White, S. G., and Spaet, T. H., Deuterohemophilia: Plasma thromboplastin factor B deficiency. Plasma thromboplastin component (PTC) deficiency, Christmas Disease, Hemophilia B. Blood, 1954, 9, 246. 